Supporting Information

\title{
Bioinspired Dual Stimuli-Responsive Membranous System with Multiple On-Off Gates
}

\author{
Bom-yi Lee ${ }^{\dagger}$, Seung Hyun ${ }^{\dagger}$, Gumhye Jeon ${ }^{\dagger}$, Eun Young $\mathrm{Kim}^{\dagger}$, Jinhwan Kim${ }^{*}$, Won-Jong \\ $\mathrm{Kim}^{+}$, and Jin Kon $\mathrm{Kim}^{\dagger *}$ \\ ${ }^{\dagger}$ National Creative Research Initiative Center for Block Copolymer Self-Assembly, \\ Department of Chemical Engineering, ${ }^{*}$ Department of Chemistry, \\ Pohang University of Science and Technology, Pohang, Kyungbuk 790-784, Korea \\ *jkkim@postech.ac.kr
}




\section{S1. Fabrication of AAO membrane}

Anodic aluminum oxide (AAO) membrane was prepared as follows. A highly pure aluminum plate $(99.999 \%, 1 \mathrm{~mm}$ thickness) was sonicated in acetone for $30 \mathrm{~min}$ and electrochemically polished twice in a polishing solution (ethanol: perchloric acid $=4$ : $1 \mathrm{v} / \mathrm{v}$ ) at $7^{\circ} \mathrm{C}$ and $20 \mathrm{~V}$ for $30 \mathrm{~min}$. Polished aluminum plate was washed with ethanol and deionized water alternately. The anodization was carried out with $0.1 \mathrm{M}$ phosphoric acid $\left(\mathrm{H}_{3} \mathrm{PO}_{4}\right)$ aqueous solution at $0{ }^{\circ} \mathrm{C}$ and $195 \mathrm{~V}$ for $18 \mathrm{~h}$, and was etched by an etchant solution ( $1.8 \mathrm{wt} \% \mathrm{H}_{2} \mathrm{CrO}_{4}$ and $6 \mathrm{wt} \% \mathrm{H}_{3} \mathrm{PO}_{4}$ in DI water) at $65{ }^{\circ} \mathrm{C}$ for over $5 \mathrm{~h}$, and washed by DI water. The pore diameter increased linearly depending on the etching time, as shown in Figure S1. In this work, we used an AAO membrane with a pore diameter of $400 \mathrm{~nm}$ and a thickness of $60 \mu \mathrm{m}$.

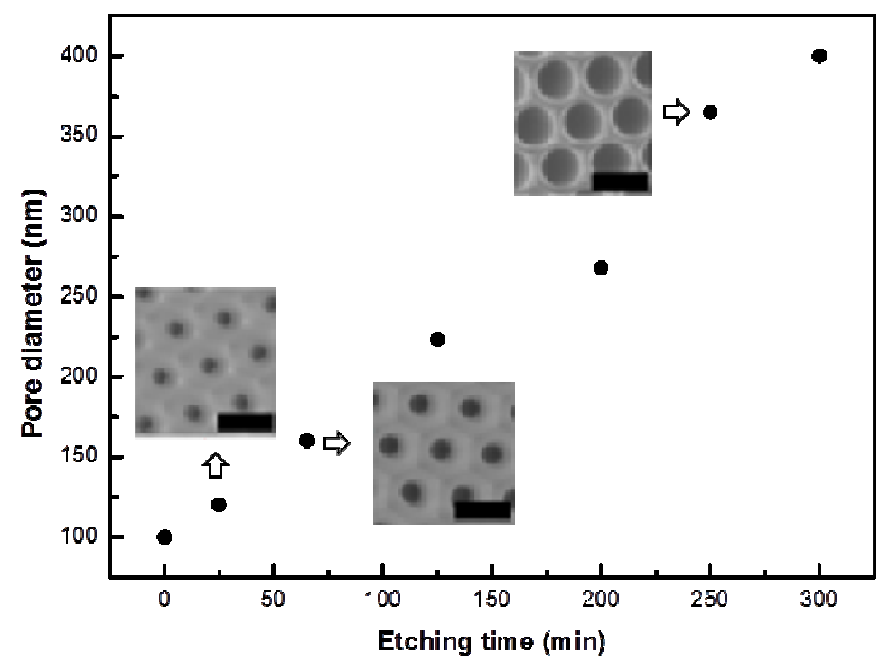

Figure S1. The change of pore diameter with etching time. 


\section{S2. Polymerization of NIPAM and AA}

Ti and gold were consecutively deposited on the top layer of AAO membrane, and this was immersed into aqueous solution of $0.1 \mathrm{M}$ cysteamine (Sigma, $\geq 98 \%$ ) for $2 \mathrm{~h}$, and cleaned with DI water. Then, the $1 \mathrm{ml}$ of 2-bromoisobutyryl bromide (BIBB) (Aldrich, $98 \%$ ) was injected dropwise into the mixture of $48 \mathrm{ml}$ of dichloromethane (DCM) (Sigma-Aldrich, anhydrous, $\geq 99.8 \%$ ) and $1.5 \mathrm{ml}$ of trimethylamine (TEA) (Sigma-Aldrich, $\geq 99 \%$ ) at $0{ }^{\circ} \mathrm{C}$ to form Br-initiated sites on the $\mathrm{Au}$ surface of the membrane. After $6 \mathrm{~h}$ reaction, the membrane was washed by DCM.

NIPAM monomer (Wako Pure Chemicals) was purified twice by recrystallization in nhexane, and dissolved it into mixture of DI water and methanol $(0.795 \mathrm{~g} / \mathrm{ml}$ of NIPAM in DI water $(31 \mathrm{ml})$ and methanol $(18 \mathrm{ml})$ ). Then, copper(I) bromide (Aldrich, $99.999 \%$ trace metals basis) (0.04 g) and copper(II) bromide (Aldrich, $99.999 \%$ trace metals basis) (0.0125 g) as a catalyst, and $\mathrm{N}, \mathrm{N}, \mathrm{N}^{\prime}, \mathrm{N}^{\prime \prime}, \mathrm{N}^{\prime \prime}$-pentamethyldiethylenetriamine (Aldrich, $\left.99 \%\right)(0.35 \mathrm{ml})$ as a ligand were added. The polymerization was carried out at $50{ }^{\circ} \mathrm{C}$ for $6 \mathrm{~h}$ under nitrogen environment. Ungrafted PNIPAM chains on AAO membrane were removed by rinsing with methanol and DI water. After drying the membrane, the contact angle of the top of AAO membrane grafted with PNIPAM chains was measured by a contact angle analyzer (300A, Surface and Electro-Optics Co.), and it was $46 \pm 2$ degrees. 
Next, the opposite side (namely, bottom side) of the membrane was consecutively deposited by $\mathrm{Ti}$ and $\mathrm{Au}$. Br-initiated sites were obtained by the same process for NIPAM monomer. Then, acrylic acid (AA) monomer (Aldrich, anhydrous, $99 \%$ ) was prepared as purified by distillation. The membrane was dipped into the $50 \mathrm{ml}$ of aqueous AA solution $(1.5 \mathrm{~mol} / \mathrm{L})$ with $\mathrm{NaOH}(\mathrm{pH}=10.2)$ at $50{ }^{\circ} \mathrm{C}$ for $8 \mathrm{~h}$ under nitrogen environment to graft AA chains on the gold layer. The copper(I) bromide $(2.97 \mathrm{mg})$ and 2,2'-bipyridine (Sigma-Aldrich, $\geq 98 \%$ ) (4.68 mg) were used as the catalyst and ligand, respectively. After AA polymerization, the membrane was cleaned with DI water three times. After drying the membrane, the contact angles of the top (PNIPAM-graft side) and bottom (PAA-grafted side) of the AAO membrane were $46 \pm 2$, and $62 \pm 3$ degrees, respectively. Since the contact angle corresponding to the upper PNIPAM side of the membrane did not change even after AAO was grafted on the bottom side of the AAO membrane, PAA polymerization only occurs at the bottom side of AAO membrane. Namely, AA does not polymerize on the top side of AAO membrane grafted with pre-existing PNIPAM chains.

Furthermore, to exclude the possibility that short PAA chains can be attached to the existing PNIPAM chains, we used Fourier transform Infrared spectroscopy (FT-IR; IFS 66V/S, Bruker optic gmbh). We prepared three samples: (1) Only PNIPAM chains are grafted, via SI-ATRP, on the AAO membrane, (2) Only PAA chains are grafted, via SI-ATRP, on the AAO membrane. (3) Additional polymerization of AA was performed on pre-existing grafted 
PNIPAM chains without additional gold deposition. It is noted the polymerization conditions of NIPAM and AA are different, as described above. Figure S2 gives FT-IR spectra of all the samples and pristine AAO membrane. Sample 1 shows PNIPAM characteristic peaks at 1646 and $1550 \mathrm{~cm}^{-1}(-\mathrm{NH}-\mathrm{C}=\mathrm{O})$ and 1388 and $1368 \mathrm{~cm}^{-1}\left(-\mathrm{CH}\left(\mathrm{CH}_{3}\right)_{2}\right)$, while sample 2 shows PAA characteristic peak at $1720 \mathrm{~cm}^{-1}(-\mathrm{C}=\mathrm{O})$. Since sample 3 does not show any characteristic peak belonging to PAA, we conclude that no PAA chain is attached to the pre-existing PNIPAM chains even after additional polymerization of AA was performed on grafted PNIPAM chains.



Figure S2. The FT-IR spectra of (a) pristine AAO membrane, (b) PNIPAM grafted AAO membrane, (c) PAA grafted AAO membrane, and (d) AAO membrane after additional polymerization of AA was performed on pre-existing grafted PNIPAM chains without additional gold deposition. 


\section{S3. Flux measurement}

\section{S3.1. Flux measurement system}

A water flux test was carried out with a home-made flux cell, as shown in Figure S3. The membrane was placed at the bottom of the flux cell, and tightly sealed by O-ring to prevent any leakage. The cell volume was $11 \mathrm{ml}$ and the total effective area of the membrane was $28.27 \mathrm{~mm}^{2}$. The temperature of the water was adjusted $\left(20\right.$ to $\left.40{ }^{\circ} \mathrm{C}\right)$ with a temperaturecontroller, while $\mathrm{pH}$ was controlled by addition of $\mathrm{HCl}$ or $\mathrm{NaOH}$. Water flows from the reservoir to the cell and passes through the membrane by nitrogen gas pressure of $0.1 \mathrm{bar}$ at a fixed temperature. The ejected water is collected at a beaker and automatically weighed with a balance at a given time interval (every 5 seconds).

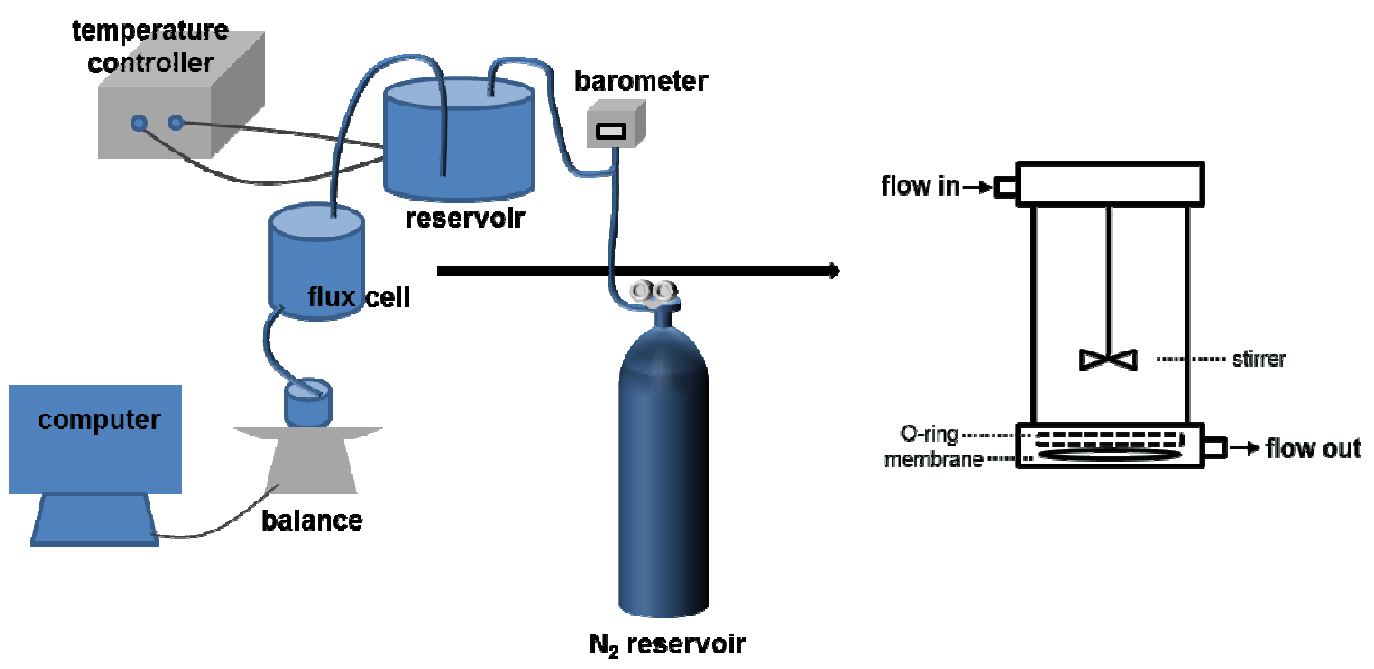

Figure S3. Schematic of flux measurement device (left) and a home-made flux cell (right). 


\section{S3.2. Flux test for the membrane grafted with only PNIPAM chains}

We performed flux test for an AAO membrane grafted with only PNIPAM chains in the upper side before PAA polymerization. Figure S4 shows the change of water flux through this membrane. At $40{ }^{\circ} \mathrm{C}$, the upper gate containing PNIPAM chains is open ("on" state). When the temperature is decreased to $20{ }^{\circ} \mathrm{C}$, the flux become zero, corresponding to "off" state, indicating that good thermo-reversibility was obtained. The water flux of this membrane is $430 \mathrm{~L} / \mathrm{m}^{2} \mathrm{~h}$, which is slightly higher than that $\left(400 \mathrm{~L} / \mathrm{m}^{2}\right)$ of the dual gated membrane grafted with PNIPAM and PAA chains in the upper and lower sides, respectively (see Figure 3 in the main text). This is because the pore diameter in the lower side of dual gated AAO membrane was reduced after PAA chains are grafted.

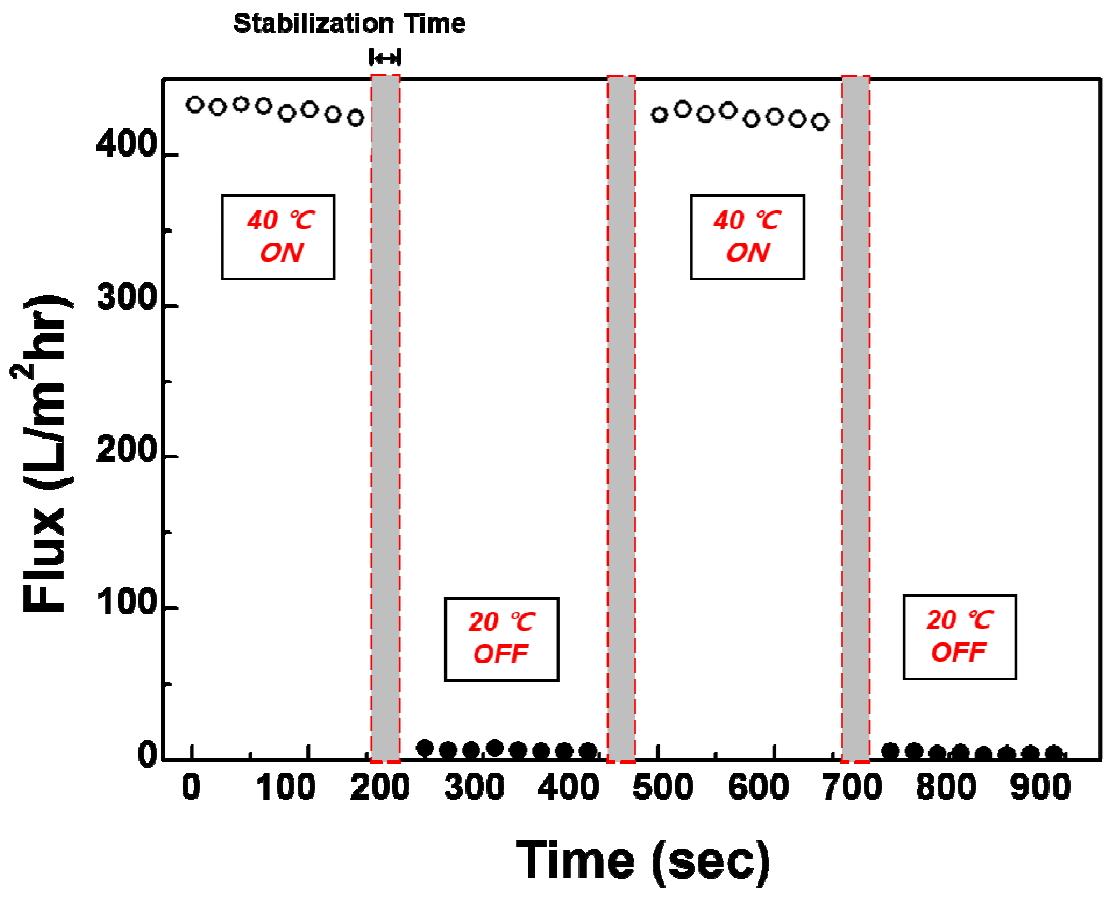

Figure S4. The change of water flux depending on temperature for an AAO membrane grafted with only PNIPAM chains in the upper side. 


\section{S4. The release of FITC-BSA}

Figure S5 shows the schematic of a home-made device for the release of fluorescein isothiocyanate-labeled bovine serum albumin (FITC-BSA) as a model biomolecule. The device consists of two chambers separated by the membrane. Each chamber contains 2/8 (vol/vol) dimethyl sulfoxide (DMSO)/water solution of $30 \mathrm{ml}$. The use of DMSO was necessary to have good dissolution of FITC-BSA into aqueous solution. The temperature of water in both chambers was controlled by water jacket. To obtain uniform temperatures inside compartments, a stirrer was placed in each chamber. Before the release text, FITCBSA was preloaded inside the pores in the membrane by immersing the membrane into DMSO/water solution with FITC-BSA for $12 \mathrm{~h}$ at $40{ }^{\circ} \mathrm{C}$ and $\mathrm{pH}=3$ corresponding to "on" and "on" gates of the membrane, which means that all pores were open. Then, the upper and lower gates in the membrane were closed by changing the solution to $20{ }^{\circ} \mathrm{C}$ and $\mathrm{pH}=6$. The membrane was immediately picked up and washed by fresh DMSO/water solution without FITC-BSA.

To prevent any leakage between two chambers, the membrane loaded with FITC-BSA was

tightened with an O-ring. Four different gating states $\left(20\right.$ and $40{ }^{\circ} \mathrm{C}$ at fixed $\mathrm{pH}=6 ; \mathrm{pH} 3$ and 6 at fixed $20{ }^{\circ} \mathrm{C}$ ) were used. The amount of released FITC-BSA inside pores of the membrane to both chambers 1 and 2 was measured by a fluorometer (Thermo scientific/ FluoroskanASCENTFL) after sampling from ports 1 and 2 at a given time interval ( $20 \mathrm{~s})$. 


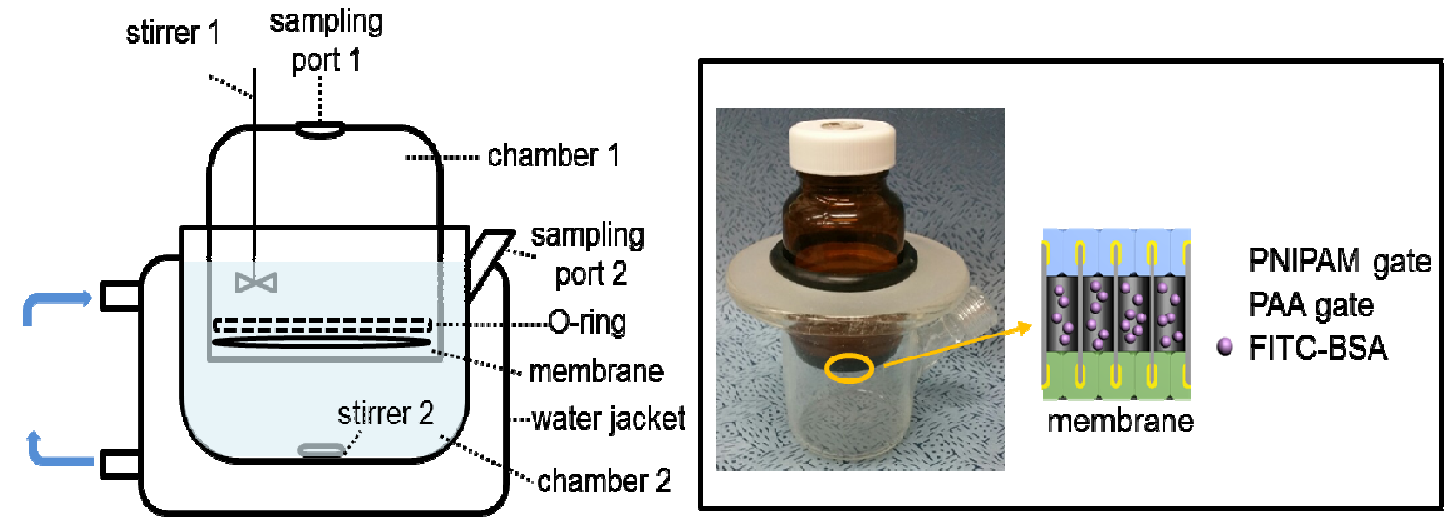

Figure S5. Schematic (left) and a real image of experimental setup for the release of

FITC-BSA inside pores of the membrane to both chambers 1 and 2 . 


\section{S5. The release of FITC-BSA by irradiation of light irradiation with two different}

wavelengths.

Since the membrane employed in this study has a gold layer, one could easily control the temperature through photothermal effect when a laser with NIR wavelength was used. Also, when an aqueous solution contains a photoacid generator, for instance, o-nitrobenzaldehyde (o-NBA), $\mathrm{pH}$ is also easily adjusted by photo irradiation with ultra-violet (UV) wavelengths. To investigate the release of FITC-BSA depending on light irradiation of these two wavelengths, we set up an experiment, as shown in Figure S6. The overall feature in Figure S6 is the same as that in Figure S5 except that there is no water jacket. Also, the lower chamber has 2/8 (vol/vol) dimethyl sulfoxide (DMSO)/water solution with o-NBA ( $5 \mathrm{wt} \%$ in solution), while the upper chamber has only $2 / 8$ (vol/vol) dimethyl sulfoxide (DMSO)/water solution. A laser for the temperature control has $808 \mathrm{~nm}$ wavelength (NIR region) at a power density of $5 \mathrm{~W} \mathrm{~cm} \mathrm{c}^{-2}$ (JENOPTIK unique-mode Gmbh, Germany), while another laser for the PH control $303 \mathrm{~nm}$ wavelength (UV region) at a power density of $4 \mathrm{~W} \mathrm{~cm}^{-2}$ (JENOPTIK unique-mode Gmbh, Germany). The laser irradiated on the top of the upper chamber. After turning on (or off) the lasers, the amount of released FITC-BSA was measured at a given interval (2s). 


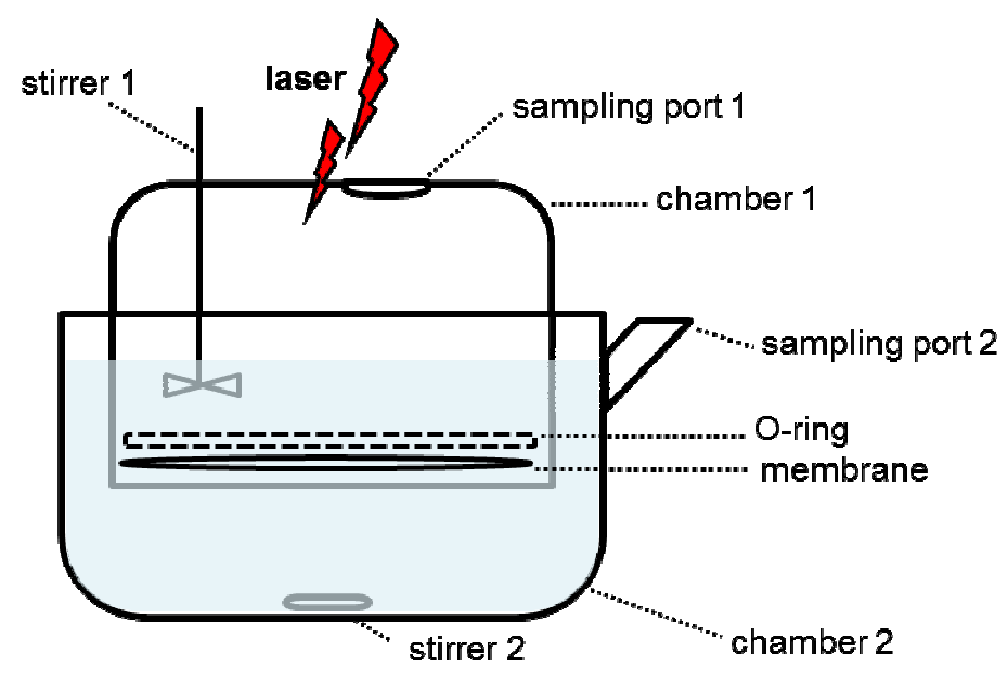

Figure S6. Schematic of experimental setup for the release of FITC-BSA inside pores of the membrane to both chambers 1 and 2 after turning on (or off) a laser. 